\title{
Quantum gravity, fakeons and microcausality
}

\author{
Damiano Anselmi and Marco Piva \\ Dipartimento di Fisica "Enrico Fermi", Università di Pisa, \\ Largo B. Pontecorvo 3, 56127 Pisa, Italy \\ INFN, Sezione di Pisa, \\ Largo B. Pontecorvo 3, 56127 Pisa, Italy \\ E-mail: damiano.anselmi@unipi.it, marco.piva@df.unipi.it
}

ABSTRACT: We investigate the properties of fakeons in quantum gravity at one loop. The theory is described by a graviton multiplet, which contains the fluctuation $h_{\mu \nu}$ of the metric, a massive scalar $\phi$ and the spin-2 fakeon $\chi_{\mu \nu}$. The fields $\phi$ and $\chi_{\mu \nu}$ are introduced explicitly at the level of the Lagrangian by means of standard procedures. We consider two options, where $\phi$ is quantized as a physical particle or a fakeon, and compute the absorptive part of the self-energy of the graviton multiplet. The width of $\chi_{\mu \nu}$, which is negative, shows that the theory predicts the violation of causality at energies larger than the fakeon mass. We address this issue and compare the results with those of the Stelle theory, where $\chi_{\mu \nu}$ is a ghost instead of a fakeon.

Keywords: Beyond Standard Model, Models of Quantum Gravity, Renormalization Regularization and Renormalons

ARXiv EPrint: 1806.03605 


\section{Contents}

1 Introduction 1

2 Isolating the fakeons in quantum gravity 3

2.1 Step 1: massive scalar 4

2.2 Step 2: spin-2 fakeon 4

3 Quantization 6

4 Absorptive part of the self-energy $\quad 9$

5 The fakeon width $\quad 12$

6 Comparison with the Stelle theory $\quad 14$

$\begin{array}{lll}7 & \text { Conclusions } & 16\end{array}$

$\begin{array}{ll}\text { A Calculations of absorptive parts } & 16\end{array}$

$\begin{array}{ll}\text { B Contributions of Proca and Pauli-Fierz fields } & 17\end{array}$

\section{Introduction}

A theory of quantum gravity was formulated in ref. [1] by means of a new prescription to treat the poles of the free propagators and turn the ghosts due to the higher derivatives into fakeons [2]. The classical Lagrangian contains the Hilbert term, the quadratic terms $\sqrt{-g} R_{\mu \nu} R^{\mu \nu}$ and $\sqrt{-g} R^{2}$ and the cosmological term. The fakeons are "fake particles", which contribute to the correlation functions, but disappear from the physical spectrum. The idea takes inspiration from the Lee-Wick models [3-6], in particular their reformulation as nonanalytically Wick rotated Euclidean theories $[7,8]$. An essentially unique ${ }^{1}$ strictly renormalizable theory of quantum gravity emerges from this approach, which is perturbatively unitary up to the effects due to the cosmological constant. ${ }^{2}$

In this paper, we investigate the properties of the fakeons in quantum gravity at one loop. To begin with, we introduce auxiliary fields and make changes of field variables, to finalize a number of arguments that are available in the literature [15] and convert the higher-derivative action of [1] into an equivalent action that does not contain higher

\footnotetext{
${ }^{1}$ This means that the action has a finite number of independent parameters and admits a finite number (two, in our case) of physically consistent quantization prescriptions.

${ }^{2} \mathrm{~A}$ consistent theory of scattering with the properties we need may not exist at nonvanishing cosmological constant. On this topic, see the discussions of refs. [9-14]. The problem concerns every realistic theory of quantum gravity, including the low-energy nonrenormalizable one, which can be used as an effective field theory.
} 
derivatives and is organized so as to fully diagonalize the kinetic part in the nonlinear case. The new setting is convenient to calculate the quantities we are interested in here. It is not equally convenient to study the renormalization of the theory (which is not affected by the fakeon prescription and has been already studied in a variety of approaches [16-22]).

Quantum gravity is described by a graviton multiplet, made of the fluctuation $h_{\mu \nu}$ of the metric tensor around flat space, a massive scalar $\phi$ and a massive spin-2 field $\chi_{\mu \nu}$. To have perturbative unitarity (up to the effects of the cosmological constant) the field $\chi_{\mu \nu}$ must be quantized as a fakeon, because its quadratic action carries the wrong overall sign. Instead, the quadratic action of $\phi$ carries the right overall sign, so $\phi$ can be quantized either as a fakeon or a physical particle. This leads to two possibilities, which we call graviton/fakeon/fakeon (GFF) theory and graviton/scalar/fakeon (GSF) theory, respectively.

We study the absorptive part of the self-energy of the graviton multiplet in both cases. A number of techniques to calculate this quantity and, more generally, deal with the fakeons, have been developed in ref. [22]. The approach we follow here further simplifies the computations and allows us to extend the results in several directions. In particular, we obtain the width $\Gamma_{\chi}$ of the spin-2 fakeon $\chi_{\mu \nu}$, which is related to the central charge $C$ of the matter fields, and the width $\Gamma_{\phi}$ of $\phi$. The value of $\Gamma_{\chi}$ is negative, which means that $\chi_{\mu \nu}$ is responsible for the violation of microcausality. At center-of-mass energies close to the fakeon mass $m_{\chi}$, and for time intervals of the order of $1 /\left|\Gamma_{\chi}\right|$ (referred to the center-of-mass frame) the common notions of past, present and future, as well as cause and effect, lose meaning. Two events can be related in a causal way only if they are separated by a time interval that is much longer than $1 /\left|\Gamma_{\chi}\right|$.

The breakdown of causality at very small distances is expected, because it is also a property of the Lee-Wick models, where it has been studied in detail [3-6, 23]. Although the quantum gravity theory of [1] is not of the Lee-Wick type, the fakeon quantization prescription introduces an infinitesimal width that turns the theory into a Lee-Wick model in an intermediate step. From the physical point of view, we do not have arguments to claim that nature must be causal up to infinite energies, so we regard the violation of microcausality as a key prediction of quantum gravity.

We also compare the results of the GFF and GSF theories with those of the Stelle theory [24], recently considered by Salvio and Strumia from the phenomenological point of view in refs. [20,21], which is a graviton/scalar/ghost (GSGh) theory. The classical action of the GSGh theory is the same, but its quantization is different in that the Feynman prescription is used for all the poles of the free propagators, including the one of $\chi_{\mu \nu}$. Then $\chi_{\mu \nu}$ is a ghost, instead of a fakeon, and does contribute to the absorptive parts, as well as the central charge $C$. The quantities we calculate do not exhibit important differences up to energies equal to the fakeon mass $m_{\chi}$. For example, the width $\Gamma_{\chi}$ is the same in the GSF and GSGh theories. The differences start to become important above $m_{\chi}$, where the optical theorem is violated in the GSGh theory.

The computations are performed at vanishing cosmological constant $\Lambda_{C}$, since the corrections due to $\Lambda_{C}$ are too small for the quantities we study. The results of ref. [22] are recovered as a particular case. We include results for Proca vectors and Pauli-Fierz spin-2 fields. 
The paper is organized as follows. In section 2 we isolate the fakeons by working out an equivalent action of quantum gravity that does not contain higher derivatives. In section 3 we outline the prescriptions to quantize the theory. In section 4 we calculate the absorptive part of the self-energy of the graviton multiplet. In section 5 we calculate the width $\Gamma_{\chi}$ of $\chi_{\mu \nu}$ and discuss the relation between $\Gamma_{\chi}$ and the central charge $C$, as well as the violations of microcausality. We also give the width of $\phi$. In section 6 we extend the calculations to the Stelle theory and compare the results with those of the GFF and GSF theories. Section 7 contains the conclusions. The appendices A and B contain details about some tools used for the calculations and other results about the absorptive parts.

\section{Isolating the fakeons in quantum gravity}

The theory of quantum gravity (coupled to matter) proposed in ref. [1] has action

$$
S_{\mathrm{QG}}=-\frac{1}{2 \kappa^{2}} \int \sqrt{-g}\left[2 \Lambda_{C}+\zeta R+\alpha\left(R_{\mu \nu} R^{\mu \nu}-\frac{1}{3} R^{2}\right)-\frac{\xi}{6} R^{2}\right]+S_{m}(g, \Phi)
$$

where $\alpha, \xi, \zeta, \Lambda_{C}$ and $\kappa$ are real constants, with $\alpha>0, \xi>0$ and $\zeta>0$, and $S_{m}$ is the action of the matter sector. For example, we can take $S_{m}$ as the covariantized action of the standard model, or one of its popular extensions, equipped with the nonminimal couplings that are compatible with the renormalizability.

In this section we isolate the fakeons by means of auxiliary fields and field redefinitions. We obtain an equivalent action that does not contain higher-derivatives and is useful for the calculations of the next sections. In particular, we fully diagonalize the kinetic part in the nonlinear case. In the next section we explain how to quantize the theory in the new setting.

To our knowledge, the new action, which is given by formula (2.10), is not available in the literature in a complete form. Partial derivations can nevertheless be found. For example, the authors of [15] work at $\Lambda_{C}=0$, with no matter sector $S_{m}$ and stop short of finalizing the action to concentrate on the analysis of the quadratic part around flat space, since their main interest is to highlight the degrees of freedom.

We assume that $S_{m}$ is at least quadratic in the matter fields $\Phi$. For simplicity, we work with bosonic fields. The arguments can be easily generalized to fermionic fields by using the tetrad formalism.

Defining

$$
\hat{\Lambda}_{C}=\Lambda_{C}\left(1+\frac{4}{3} \frac{\xi \Lambda_{C}}{\zeta^{2}}\right), \quad \hat{\zeta}=\zeta \frac{\hat{\Lambda}_{C}}{\Lambda_{C}}, \quad \hat{R}_{\mu \nu}=R_{\mu \nu}+\frac{\Lambda_{C}}{\zeta} g_{\mu \nu}, \quad \hat{R}=R+\frac{4 \Lambda_{C}}{\zeta},
$$

and adding the integral of a total derivative, the action (2.1) can be written in the more convenient form

$$
S_{\mathrm{QG}}=\hat{S}_{\mathrm{HE}}(g)+S_{\mathrm{W}}(g)+\frac{\xi}{12 \kappa^{2}} \int \sqrt{-g} \hat{R}^{2}+S_{m}(g, \Phi),
$$

where

$$
\hat{S}_{\mathrm{HE}}(g)=-\frac{1}{2 \kappa^{2}} \int \sqrt{-g}\left(2 \hat{\Lambda}_{C}+\hat{\zeta} R\right)
$$


is the Hilbert-Einstein action and

$$
S_{\mathrm{W}}(g)=-\frac{\alpha}{4 \kappa^{2}} \int \sqrt{-g} C_{\mu \nu \rho \sigma} C^{\mu \nu \rho \sigma}
$$

is the Weyl action, $C_{\mu \nu \rho \sigma}$ denoting the Weyl tensor.

\section{$2.1 \quad$ Step 1: massive scalar}

We introduce an auxiliary field $\hat{\phi}$ and write $S_{\mathrm{QG}}$ as

$$
S_{\mathrm{QG}}=\hat{S}_{\mathrm{HE}}(g)+S_{\mathrm{W}}(g)+\frac{\xi}{12 \kappa^{2}} \int \sqrt{-g}(2 \hat{R}-\hat{\phi}) \hat{\phi}+S_{m}(g, \Phi) .
$$

Then we perform the Weyl transformation

$$
g_{\mu \nu} \rightarrow g_{\mu \nu} \mathrm{e}^{\kappa \phi},
$$

where

$$
\phi=-\frac{1}{\kappa} \ln \left(1-\frac{\xi \hat{\phi}}{3 \hat{\zeta}}\right) .
$$

So doing, we obtain the equivalent action

$$
S_{\mathrm{QG}}=\hat{S}_{\mathrm{HE}}(g)+S_{\mathrm{W}}(g)+S_{\phi}(g, \phi)+S_{m}\left(g \mathrm{e}^{\kappa \phi}, \Phi\right),
$$

where

$$
S_{\phi}(g, \phi)=\frac{3 \hat{\zeta}}{4} \int \sqrt{-g}\left[D_{\mu} \phi D^{\mu} \phi-\frac{m_{\phi}^{2}}{\kappa^{2}}\left(1-\mathrm{e}^{\kappa \phi}\right)^{2}\right],
$$

the squared mass of $\phi$ being

$$
m_{\phi}^{2}=\frac{\zeta}{\xi}
$$

\subsection{Step 2: spin-2 fakeon}

Now we take care of the spin-2 fakeon. We have

$$
\hat{S}_{\mathrm{HE}}(g)+S_{\mathrm{W}}(g)=\tilde{S}_{\mathrm{HE}}(g)-\frac{\alpha}{2 \kappa^{2}} \int \sqrt{-g}\left(\tilde{R}_{\mu \nu} \tilde{R}^{\mu \nu}-\frac{1}{3} \tilde{R}^{2}\right),
$$

up to the integral of a total derivative, where

$$
\begin{array}{rlrl}
\tilde{S}_{\mathrm{HE}}(g) & =-\frac{1}{2 \kappa^{2}} \int \sqrt{-g}\left(2 \tilde{\Lambda}_{C}+\tilde{\zeta} R\right), & \tilde{R}_{\mu \nu}=\hat{R}_{\mu \nu}, \\
\tilde{\Lambda}_{C}=\hat{\Lambda}_{C}\left(1+\frac{2}{3} \frac{\alpha \hat{\Lambda}_{C}}{\hat{\zeta}^{2}}\right)=\Lambda_{C}\left(1+\frac{2}{3} \frac{(\alpha+2 \xi) \Lambda_{C}}{\zeta^{2}}\right), & \tilde{\zeta}=\hat{\zeta} \frac{\tilde{\Lambda}_{C}}{\hat{\Lambda}_{C}}=\zeta \frac{\tilde{\Lambda}_{C}}{\Lambda_{C}} .
\end{array}
$$

We introduce auxiliary fields $\chi_{\mu \nu}$ by writing the action $S_{\mathrm{QG}}$ as

$$
\begin{aligned}
S_{\mathrm{QG}}= & \tilde{S}_{\mathrm{HE}}(g)-\frac{\tilde{\zeta}}{2 \kappa^{2}} \int \sqrt{-g}\left[2 \chi^{\mu \nu}\left(\tilde{R}_{\mu \nu}-\frac{1}{2} g_{\mu \nu} \tilde{R}\right)-\frac{\tilde{\zeta}}{\alpha}\left(\chi_{\mu \nu} \chi^{\mu \nu}-\chi^{2}\right)\right] \\
& +S_{\phi}(g, \phi)+S_{m}\left(g \mathrm{e}^{\kappa \phi}, \Phi\right),
\end{aligned}
$$


where $\chi=\chi_{\mu \nu} g^{\mu \nu}$. At this point, we perform the metric-tensor redefinition

$$
g_{\mu \nu} \rightarrow g_{\mu \nu}+2 \chi_{\mu \nu}+\chi_{\mu \nu} \chi-2 \chi_{\mu \rho} \chi_{\nu}^{\rho} \equiv g_{\mu \nu}+\psi_{\mu \nu} .
$$

The linear contribution to $\psi_{\mu \nu}$ is fixed so that the transformed action contains no terms that are linear in $\chi_{\mu \nu}$. The quadratic corrections are determined so that the mass terms of the $\chi_{\mu \nu}$ action get the right Pauli-Fierz form and the limit $\Lambda_{C} \rightarrow 0$ remains regular.

Applying the redefinition (2.9) to (2.8), we obtain the equivalent action of quantum gravity we are going to work with in this paper, which reads

$$
S_{\mathrm{QG}}(g, \phi, \chi, \Phi)=\tilde{S}_{\mathrm{HE}}(g)+S_{\chi}(g, \chi)+S_{\phi}(g+\psi, \phi)+S_{m}\left(g \mathrm{e}^{\kappa \phi}+\psi \mathrm{e}^{\kappa \phi}, \Phi\right),
$$

where

$$
S_{\chi}(g, \chi)=\tilde{S}_{\mathrm{HE}}(g+\psi)-\tilde{S}_{\mathrm{HE}}(g)+\int\left[-2 \chi_{\mu \nu} \frac{\delta \tilde{S}_{\mathrm{HE}}(g)}{\delta g_{\mu \nu}}+\frac{\tilde{\zeta}^{2}}{2 \alpha \kappa^{2}} \sqrt{-g}\left(\chi_{\mu \nu} \chi^{\mu \nu}-\chi^{2}\right)\right]_{g \rightarrow g+\psi}
$$

is the action of the fakeon $\chi_{\mu \nu}$. We find

$$
S_{\chi}(g, \chi)=-\frac{\tilde{\zeta}}{\kappa^{2}} S_{\mathrm{PF}}\left(g, \chi, m_{\chi}^{2}\right)-\frac{\tilde{\zeta}}{2 \kappa^{2}} \int \sqrt{-g} R^{\mu \nu}\left(\chi \chi_{\mu \nu}-2 \chi_{\mu \rho} \chi_{\nu}^{\rho}\right)+S_{\chi}^{(>2)}(g, \chi),
$$

where

$$
\begin{aligned}
S_{\mathrm{PF}}\left(g, \chi, m_{\chi}^{2}\right)= & \frac{1}{2} \int \sqrt{-g}\left[D_{\rho} \chi_{\mu \nu} D^{\rho} \chi^{\mu \nu}-D_{\rho} \chi D^{\rho} \chi+2 D_{\mu} \chi^{\mu \nu} D_{\nu} \chi-2 D_{\mu} \chi^{\rho \nu} D_{\rho} \chi_{\nu}^{\mu}\right. \\
& \left.-m_{\chi}^{2}\left(\chi_{\mu \nu} \chi^{\mu \nu}-\chi^{2}\right)\right]
\end{aligned}
$$

is the covariantized Pauli-Fierz action and $S_{\chi}^{(>2)}(g, \chi)$ are corrections that are at least cubic in $\chi$. The squared mass of the spin- 2 fakeon is

$$
m_{\chi}^{2}=\frac{\tilde{\zeta}}{\alpha}
$$

The transformations (2.3), (2.4) and (2.9) are ultralocal (i.e. they depend on the fields, but not their derivatives), so the Jacobians are identically one in dimensional regularization. This means that we can use the new action $S_{\mathrm{QG}}(g, \phi, \chi, \Phi)$ of formula (2.10) as the action of quantum gravity at the level of the functional integral.

So far, we have kept the cosmological constant different from zero, but in many situations it may be neglected. When that is the case, it is convenient to replace the field redefinition (2.9) with

$$
g_{\mu \nu} \rightarrow g_{\mu \nu}+2 \chi_{\mu \nu}
$$

so that, instead of (2.10), we have

$$
S_{\mathrm{QG}}(g, \phi, \chi, \Phi)=S_{\mathrm{H}}(g)+S_{\chi}^{\prime}(g, \chi)+S_{\phi}(g+2 \chi, \phi)+S_{m}\left(g \mathrm{e}^{\kappa \phi}+2 \chi \mathrm{e}^{\kappa \phi}, \Phi\right),
$$


where

$$
S_{\mathrm{H}}(g)=-\frac{\zeta}{2 \kappa^{2}} \int \sqrt{-g} R,
$$

is the Hilbert action and $S_{\chi}^{\prime}(g, \chi)$ is the new $\chi$ action, still given by $(2.11)$, but with $\Lambda_{C}=0$ and $\psi_{\mu \nu}$ replaced by $2 \chi_{\mu \nu}$. We find

$$
\begin{aligned}
S_{\chi}^{\prime}(g, \chi)= & -2 \int \frac{\delta^{2} S_{\mathrm{H}}}{\delta g_{\mu \nu}(x) \delta g_{\rho \sigma}(y)} \chi_{\mu \nu}(x) \chi_{\rho \sigma}(y) \mathrm{d} x \mathrm{~d} y+\frac{\zeta^{2}}{2 \alpha \kappa^{2}} \int \sqrt{-g}\left(\chi_{\mu \nu} \chi^{\mu \nu}-\chi^{2}\right) \\
& -\frac{8}{3} \int \frac{\delta^{3} S_{\mathrm{H}}}{\delta g_{\mu \nu}(x) \delta g_{\rho \sigma}(y) \delta g_{\alpha \beta}(z)} \chi_{\mu \nu}(x) \chi_{\rho \sigma}(y) \chi_{\alpha \beta}(z) \mathrm{d} x \mathrm{~d} y \mathrm{~d} z \\
& +\frac{\zeta^{2}}{2 \alpha \kappa^{2}} \int \sqrt{-g}\left(5 \chi \chi_{\mu \nu} \chi^{\mu \nu}-4 \chi_{\mu \nu} \chi^{\mu \rho} \chi_{\rho}^{\nu}-\chi^{3}\right)+S_{\chi}^{(>3)}(g, \chi) .
\end{aligned}
$$

where $S_{\chi}^{(>3)}(g, \chi)$ are corrections that are at least quartic in $\chi_{\mu \nu}$, which are not needed in the calculations of this paper. Note that the nonminimal couplings of the quadratic part

$$
-\frac{\zeta}{\kappa^{2}} S_{\mathrm{PF}}\left(g, \chi, m_{\chi}^{2}\right)-\frac{\zeta}{4 \kappa^{2}} \int \sqrt{-g}\left(4 \chi \chi_{\mu \nu} R^{\mu \nu}-8 \chi_{\mu \nu} \chi^{\nu \rho} R_{\rho}^{\mu}+2 R \chi_{\mu \nu} \chi^{\mu \nu}-R \chi^{2}\right)
$$

of $S_{\chi}^{\prime}(g, \chi)$ differ from those of (2.12), and the $\chi$ squared mass is now

$$
m_{\chi}^{2}=\frac{\zeta}{\alpha}
$$

Formulas (2.11) and (2.17) show that the vertices of the $\chi$ actions are related to the vertices of the Hilbert-Einstein action, apart from corrections proportional to $m_{\chi}^{2}$.

The new actions (2.10) and (2.16) are convenient to calculate the quantities we are interested in, but make the renormalizability of the theory much less evident than it was in the original field variables (2.1). On general grounds, the only effect of a perturbative change of field variables on the divergent sector of the theory is to require extra field renormalizations, which are generically nonpolynomial, yet perturbatively local. A precise match between the divergent parts, calculated before and after the field redefinition, can be worked out by relating them to the renormalizations of the composite operators involved in the transformation [25-27].

\section{Quantization}

Expanding the metric tensor around flat space as $g_{\mu \nu}=\eta_{\mu \nu}+2 \kappa h_{\mu \nu}$, where $\eta_{\mu \nu}=\operatorname{diag}(1$, $-1,-1,-1)$, the graviton sector is described by the graviton multiplet

$$
G_{A}=\left\{h_{\mu \nu}, \phi, \chi_{\rho \sigma}\right\}
$$

made of the fluctuation $h_{\mu \nu}$ of the metric, the massive scalar $\phi$ and the massive spin-2 field $\chi_{\mu \nu}$.

Assuming that $\left|\Lambda_{C}\right|$ is sufficiently small, so that both $\tilde{\zeta}$ and $\hat{\zeta}$ are positive, the action $S_{\chi}$ of formula (2.12) carries the wrong overall sign. This means that, to have perturbative unitarity (up to corrections due to the cosmological constant), $\chi_{\mu \nu}$ must be quantized as a 
fakeon, following the prescription of ref. [1]. Instead, the quadratic action $S_{\phi}$ of eq. (2.6) carries the right overall sign, so $\phi$ can be quantized either as a fakeon or a physical particle. This leads to two possibilities, which we call graviton/fakeon/fakeon (GFF) theory and graviton/scalar/fakeon (GSF) theory, respectively. Being perturbatively unitary (up to the effects of the cosmological constant) and renormalizable, they are both good candidates to describe quantum gravity. We could also view $\phi$ and $\chi_{\mu \nu}$ as part of the matter sector.

We define the GFF and GSF prescriptions by introducing two infinitesimal widths $\epsilon$ and $\mathcal{E}$ in the propagators as follows:

(a) replace $p^{2}$ with $p^{2}+i \epsilon$ everywhere in the denominators of the propagators, where $p$ denotes the momentum;

(b) turn the $\chi$ poles into fakeons by means of the replacement

$$
\frac{1}{p^{2}-m_{\chi}^{2}+i \epsilon} \rightarrow \frac{p^{2}-m_{\chi}^{2}}{\left(p^{2}-m_{\chi}^{2}+i \epsilon\right)^{2}+\mathcal{E}^{4}}
$$

(c) [only in the GFF case] turn the $\phi$ poles into fakeons by means of the replacement

$$
\frac{1}{p^{2}-m_{\phi}^{2}+i \epsilon} \rightarrow \frac{p^{2}-m_{\phi}^{2}}{\left(p^{2}-m_{\phi}^{2}+i \epsilon\right)^{2}+\mathcal{E}^{4}} .
$$

(d) calculate the diagrams in the Euclidean framework, nonanalytically Wick rotate them as explained in refs. $[2,7,8]$, then make $\epsilon$ tend to zero first and $\mathcal{E}$ tend to zero last.

Note that because of the Wick rotation involved in point $(d)$ the distributions appearing on the right-hand sides of eqs. (3.2) and (3.3) do not give the principal value (which would require to integrate on real energies).

An equivalent, and often more efficient, way to formulate the graviton/fakeon prescription is to combine point $(a)$ with the requirement that, in evaluating the loop integrals,

$\left(a^{\prime}\right)$ every threshold involving a fakeon must be overcome by means of the average continuation, which is the arithmetic average of the two analytic continuations that circumvent the threshold.

The space of the complexified external momenta is divided into disjoint regions of analyticity. All of them can be unambiguously reached from the Euclidean region by means of the average continuation.

The free propagator of the metric fluctuation $h_{\mu \nu}$ reads

$$
\left\langle h_{\mu \nu}(p) h_{\rho \sigma}(-p)\right\rangle_{0}=\frac{i\left(\eta_{\mu \rho} \eta_{\nu \sigma}+\eta_{\mu \sigma} \eta_{\nu \rho}-\eta_{\mu \nu} \eta_{\rho \sigma}\right)}{2 \tilde{\zeta}\left(p^{2}-m_{h}^{2}+i \epsilon\right)},
$$

where $m_{h}^{2}=-2 \Lambda_{C} / \zeta$, in the de Donder gauge. We recall that the cutting equations [28, 29] (which are the diagrammatic equations that lead to the optical theorem) are formally satisfied even when cosmological constant is nonvanishing, as long as it is negative [1], although a consistent theory of scattering likely does not exist in that case. 
The free propagator of $\chi_{\mu \nu}$ reads

$$
\left\langle\chi_{\mu \nu}(p) \chi_{\rho \sigma}(-p)\right\rangle_{0}=-\frac{i \kappa^{2}}{\tilde{\zeta}} \frac{p^{2}-m_{\chi}^{2}}{\left(p^{2}-m_{\chi}^{2}+i \epsilon\right)^{2}+\mathcal{E}^{4}} \prod_{\mu \nu \rho \sigma}^{(2)}\left(p, m_{\chi}^{2}\right)
$$

where

$$
\prod_{\mu \nu \rho \sigma}^{(2)}\left(p, m_{\chi}^{2}\right)=\frac{1}{2}\left(\pi_{\mu \rho} \pi_{\nu \sigma}+\pi_{\mu \sigma} \pi_{\nu \rho}-\frac{2}{3} \pi_{\mu \nu} \pi_{\rho \sigma}\right), \quad \pi_{\mu \nu}=\eta_{\mu \nu}-\frac{p_{\mu} p_{\nu}}{m_{\chi}^{2}}
$$

are spin-2 and spin- 1 on-shell projectors, respectively.

The free $\phi$ propagator reads

$$
\langle\phi(p) \phi(-p)\rangle_{0 \mathrm{GFF}}=\frac{2 i}{3 \hat{\zeta}} \frac{p^{2}-m_{\phi}^{2}}{\left(p^{2}-m_{\phi}^{2}+i \epsilon\right)^{2}+\mathcal{E}^{4}}, \quad\langle\phi(p) \phi(-p)\rangle_{0 \mathrm{GSF}}=\frac{2 i}{3 \hat{\zeta}} \frac{1}{p^{2}-m_{\phi}^{2}+i \epsilon},
$$

in the GFF and GSF cases, respectively.

The physical fields are the physical components of $h_{\mu \nu}$ (obtained by projecting away the unphysical components and the Faddeev-Popov ghosts in the usual ways), the massive scalar $\phi$ (in the GSF theory only) and the matter fields $\Phi$.

The Fock space $V$ of the physical states is the Hilbert space built as follows. Consider the states $|n\rangle$ obtained by acting on the vacuum $|0\rangle$ by means of the creation operators of the physical fields. Then, build the metric space $\mathcal{F}$ made of the finite linear combinations of the states $|n\rangle$. Finally, complete $\mathcal{F}$ to the Hilbert space $V$ by means of the Cauchy procedure.

The space $V$ is a proper subspace of the total Fock space $W$, which also contains the states built with the creation operators of the fakeons ( $a_{\chi}^{\dagger}$ in the GSF theory and $a_{\chi}^{\dagger}, a_{\phi}^{\dagger}$ in the GFF theory). The free Hamiltonian $H_{\text {free }}$ is bounded from below in $V$, although it is not bounded from below in $W$ (due to the negative contributions brought by $\chi_{\mu \nu}$ ). Perturbative unitarity is the statement that the projection from $W$ onto $V$ is consistent, i.e. the states that are projected away are not generated back in the cutting equations and the optical theorem. More details are given in sections 5 and 6 .

Before turning to the computations, let us recall that the standard quantization prescription [24] is just made of point ( $a$ ) for every pole. Then $\phi$ is a physical particle, but $\chi_{\mu \nu}$ is a ghost, due to the overall minus sign that multiplies the right-hand side of (3.5). In that case the Fock space is the whole $W$.

Another interesting possibility has been pointed out by Avramidi and Barvinsky in ref. [18], where it was noted that for $\Lambda_{C}>0, \xi<0$ the action (2.1) is positive definite in the Euclidean framework and the theory is asymptotically free (when matter is switched off). However, $\xi<0$ makes the squared mass of $\phi$ negative. The fakeon prescription of ref. [1] works for poles located on the real axis, irrespectively of the sign of the residue at the pole. Tachyons do not fall in that class, so we cannot guarantee in this moment that a proper generalization of the prescription (3.3) exists for $\xi<0$. 


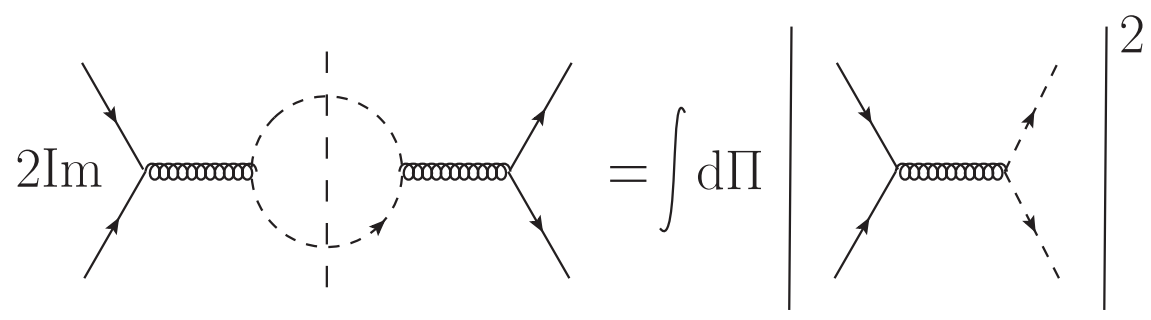

Figure 1. Processes involving the absorptive part of the graviton-multiplet self-energy.

\section{Absorptive part of the self-energy}

The absorptive part of the self-energy of the graviton multiplet is important because it allows us to extract physically observable quantities, as explained in section 5 . In figure 1 we show a basic process where the absorptive part plays a key role. On the right-hand side, we have the squared modulus of the transition amplitude between some initial states, denoted by the continuous lines, and some final states, denoted by the dashed lines. The wiggled line denotes the graviton multiplet. Integrating on the phase space $\Pi$ of the final states, we obtain twice the imaginary part of the amplitude shown on the left-hand side. In this section, we ignore the initial states, which leads us to consider the absorptive parts

$$
M_{A B} \equiv\left\langle G_{A}(p) G_{B}(-p)\right\rangle_{\text {abs }}^{1 \text { loop }}
$$

of the matrix $\left\langle G_{A} G_{B}\right\rangle$ of the graviton-multiplet two-point functions at one loop. For simplicity, we set the cosmological constant to zero, but the procedure can be easily generalized to $\Lambda_{C} \neq 0$. The gauge-dependent contributions are calculated in the de Donder gauge.

We can throw away the diagrams where the fakeons propagate inside the loop. Indeed, according to the prescription of the previous section, in those cases we are lead to calculate the average continuation above the thresholds, which has no absorptive part. Then we can drop $S_{\chi}^{\prime}(g, \chi)$ from the action $(2.16)$ and work with the simplified action

$$
S_{\mathrm{QG}}^{\prime}(g, \phi, \chi, \Phi)=S_{\mathrm{H}}(g)+S_{\phi}(g+2 \chi, \phi)+S_{m}\left(g \mathrm{e}^{\kappa \phi}+2 \chi \mathrm{e}^{\kappa \phi}, \Phi\right) .
$$

The tadpole diagrams do not contribute to the absorptive parts, so we can focus on the cubic vertices. Expanding (4.2) to the cubic order in $\chi_{\mu \nu}-\phi-\Phi$, we obtain a further simplified action for the GSF theory, which is

$$
\begin{aligned}
S_{\mathrm{QG}}^{\mathrm{GSF}}(g, \phi, \chi, \Phi)= & S_{\mathrm{H}}(g)+S_{\phi}(g, \phi)+S_{m}(g, \Phi) \\
& -\frac{1}{2} \int \sqrt{-g}\left[\left(2 \chi_{\mu \nu}+\kappa \phi g_{\mu \nu}\right) T_{m}^{\mu \nu}(g, \Phi)+2 \chi_{\mu \nu} T_{\phi}^{\mu \nu}(g, \phi)\right],
\end{aligned}
$$

where

$$
T_{m}^{\mu \nu}(g, \Phi)=-\frac{2}{\sqrt{-g}} \frac{\delta S_{m}(g, \Phi)}{\delta g_{\mu \nu}}, \quad T_{\phi}^{\mu \nu}(g, \phi)=-\frac{2}{\sqrt{-g}} \frac{\delta S_{\phi}(g, \phi)}{\delta g_{\mu \nu}},
$$

are the energy-momentum tensor of the matter fields and the one of $\phi$, respectively. 
In the GFF case, the field $\phi$ can also be ignored inside the loop, which means that we can work with

$$
S_{\mathrm{QG}}^{\mathrm{GFF}}(g, \phi, \chi, \Phi)=S_{\mathrm{H}}(g)+S_{m}(g, \Phi)-\frac{1}{2} \int \sqrt{-g}\left(2 \chi_{\mu \nu}+g_{\mu \nu} \kappa \phi\right) T_{m}^{\mu \nu}(g, \Phi) .
$$

We collect the results about $M_{A B}$ into the one-loop absorptive part $\Gamma_{\text {abs }}$ of the $\Gamma$ functional. We can decompose $\Gamma_{\text {abs }}$ as

$$
\begin{aligned}
\Gamma_{\mathrm{abs}}^{\mathrm{GFF}} & =\Gamma_{\mathrm{abs}}^{h h}+\Gamma_{\mathrm{abs}}^{m}, \\
\Gamma_{\mathrm{abs}}^{\mathrm{GSF}} & =\Gamma_{\mathrm{abs}}^{h h}+\Gamma_{\mathrm{abs}}^{\phi h}+\Gamma_{\mathrm{abs}}^{\phi \phi}+\Gamma_{\mathrm{abs}}^{m},
\end{aligned}
$$

in the GFF and GSF cases, respectively, where $\Gamma_{\text {abs }}^{h h}$ includes the contributions of the $h$ bubble and the bubble of Faddeev-Popov ghosts, while in the other cases the fields circulating in the loop are specified by the superscripts.

The contributions $\Gamma_{\mathrm{abs}}^{h h}$ are gauge dependent and can be collected into field redefinitions, up to cubic corrections (which do not contribute to $M_{A B}$ ). Their expressions can be read from formulas (4.8)-(4.9) of ref. [22] in the limit $\alpha \rightarrow 0, \xi \rightarrow 0$. The result is

$$
\Gamma_{\mathrm{abs}}^{h h}=-\int \frac{\delta S_{\mathrm{H}}(g)}{\delta g_{\mu \nu}} \Delta g_{\mu \nu}, \quad \Delta g_{\mu \nu}=\frac{i \kappa^{3}}{480 \pi \zeta} \theta(-\square)\left(61 \square h_{\mu \nu}-42 \eta_{\mu \nu} \square h+42 \eta_{\mu \nu} \partial^{\rho} \partial^{\sigma} h_{\rho \sigma}\right) .
$$

Formula (4.8) can be rewritten as

$$
\Gamma_{\mathrm{abs}}^{h h}=-\int \frac{\delta S_{\mathrm{QG}}}{\delta g_{\mu \nu}} \Delta g_{\mu \nu}
$$

up to cubic corrections.

The contributions $\Gamma_{\text {abs }}^{\phi h}$ are also gauge dependent away from the $\phi$ peak. They can be calculated with the techniques explained in appendix A. We find

$$
\Gamma_{\mathrm{abs}}^{\phi h}=-\frac{3 i \kappa^{2} m_{\phi}^{2}}{32 \pi} \int \sqrt{-g} \phi \theta\left(-\square-m_{\phi}^{2}\right)\left(\square+m_{\phi}^{2}\right) \frac{1}{\square}\left(2 \square+m_{\phi}^{2}\right) \phi .
$$

The other contributions to $\Gamma_{\text {abs }}$ are gauge independent. We find

$$
\begin{aligned}
\Gamma_{\mathrm{abs}}^{\phi \phi}= & \left.\frac{i}{16 \pi} \frac{1}{120} \int \sqrt{-g} R_{\mu \nu} \theta(r) \theta(1-r) \sqrt{1-r}\left[(1-r)^{2} R^{\mu \nu}+\frac{1}{8}\left(4+12 r-r^{2}\right) g^{\mu \nu} R\right]\right|_{g \rightarrow g+2 \chi} \\
& +\left.\frac{i \kappa}{512 \pi} \int \sqrt{-g} \phi \theta(r) \theta(1-r) \sqrt{1-r}\left[72 m_{\phi}^{2} \kappa \phi-r(2+r) \square R\right]\right|_{g \rightarrow g+2 \chi}
\end{aligned}
$$

where $r=-4 m_{\phi}^{2} / \square$ and the substitutions $g \rightarrow g+2 \chi$ are to be performed on the whole integrands.

Now we turn to $\Gamma_{\mathrm{abs}}^{m}$. Equation (4.3) shows that these corrections are related to the two-point function of the energy-momentum tensor $T_{m}^{\mu \nu}$ of equation (4.4). We can write

$$
\Gamma_{\mathrm{abs}}^{m}=\Gamma_{\mathrm{abs}}^{\varphi}+\Gamma_{\mathrm{abs}}^{\psi}+\Gamma_{\mathrm{abs}}^{V},
$$

where $\Gamma_{\mathrm{abs}}^{\varphi}, \Gamma_{\mathrm{abs}}^{\psi}$ and $\Gamma_{\text {abs }}^{V}$ are the contributions of scalar fields, fermions and gauge vectors, respectively. 
On general grounds, the matter fields $\Phi$ of mass $m_{\Phi}$ give an expression of the form

$$
\begin{aligned}
\Gamma_{\mathrm{abs}}^{\Phi}= & \frac{i}{16 \pi} \int \sqrt{-g} R_{\mu \nu} \theta\left(r_{\Phi}\right) \theta\left(1-r_{\Phi}\right) \sqrt{1-r_{\Phi}} \\
& \times\left.\left[P_{\Phi}\left(r_{\Phi}\right)\left(R^{\mu \nu}-\frac{1}{3} g^{\mu \nu} R\right)+Q_{\Phi}\left(r_{\Phi}\right) g^{\mu \nu} R\right]\right|_{g \rightarrow(g+2 \chi) \mathrm{e}^{\kappa \phi}},
\end{aligned}
$$

where $r_{\Phi}=-4 m_{\Phi}^{2} / \square$ and $P_{\Phi}\left(r_{\Phi}\right), Q_{\Phi}\left(r_{\Phi}\right)$ are polynomials that can be calculated as explained in appendix A.

In the case of $N_{s}$ scalar fields of mass $m_{\varphi}$, with action

$$
S_{s}=\frac{1}{2} \sum_{i=1}^{N_{s}} \int \sqrt{-g}\left[g^{\mu \nu}\left(\partial_{\mu} \varphi^{i}\right)\left(\partial_{\nu} \varphi^{i}\right)-m_{\varphi}^{2} \varphi^{i 2}+\frac{1}{6}\left(1+2 \eta_{s}\right) R \varphi^{i 2}\right],
$$

we find that $\Gamma_{\text {abs }}^{\varphi}$ is given by formula (4.12) with

$$
P_{\varphi}(r)=\frac{N_{s}}{120}(1-r)^{2}, \quad Q_{\varphi}(r)=\frac{N_{s}}{576}\left(4 \eta_{s}-r\right)^{2} .
$$

In the case of $N_{f}$ Dirac fermions of mass $m_{\psi}, \Gamma_{\text {abs }}^{\psi}$ is given by the same formula with

$$
P_{\psi}(r)=\frac{N_{f}}{60}\left(3-r-2 r^{2}\right), \quad Q_{\psi}(r)=\frac{N_{f}}{144} r(1-r) .
$$

In the case of $N_{v}$ gauge vectors $V_{\mu}, \Gamma_{\text {abs }}^{V}$ is given by

$$
P_{V}(0)=\frac{N_{v}}{10}, \quad Q_{V}(0)=0 .
$$

For completeness, we also consider Proca vectors $A_{\mu}$ and Pauli-Fierz symmetric tensors $\Upsilon_{\mu \nu}$. The Proca action is

$$
S_{\mathrm{P}}(g, A)=\int \sqrt{-g}\left[-\frac{1}{4} F_{\mu \nu} F^{\mu \nu}+\frac{m_{\mathrm{P}}^{2}}{2} A^{\mu} A_{\mu}+\frac{\eta_{\mathrm{P}}}{2} R^{\mu \nu} A_{\mu} A_{\nu}+\frac{\eta_{\mathrm{P}}^{\prime}}{2} R A^{\mu} A_{\mu}\right],
$$

where $\eta_{\mathrm{P}}$ and $\eta_{\mathrm{P}}^{\prime}$ parametrize the nonminimal couplings. The contribution $\Gamma_{\mathrm{abs}}^{\mathrm{P}}$ of $N_{\mathrm{P}}$ copies of such vectors to the absorptive part is (4.12) with

$$
P_{\mathrm{P}}(r)=\frac{N_{\mathrm{P}}}{120}\left(13+14 r+3 r^{2}\right)+P_{\mathrm{P}}^{\mathrm{nm}}(r), \quad Q_{\mathrm{P}}(r)=\frac{N_{\mathrm{P}}}{576}\left(4-4 r+3 r^{2}\right)+Q_{\mathrm{P}}^{\mathrm{nm}}(r),
$$

where $P_{\mathrm{P}}^{\mathrm{nm}}(r)$ and $Q_{\mathrm{P}}^{\mathrm{nm}}(r)$ are corrections due to the nonminimal couplings, collected in appendix B. A curious fact is that $\Gamma_{\mathrm{abs}}^{\mathrm{P}}$ admits a regular ultraviolet limit $\left(m_{\mathrm{P}} \rightarrow 0\right)$ at $\eta_{\mathrm{P}}=\eta_{\mathrm{P}}^{\prime}=0$. However, the limit is not conformal invariant, since $Q_{\mathrm{P}}(0) \neq 0$, due to simplifications between powers of $m_{\mathrm{P}}$ and powers of $1 / m_{\mathrm{P}}$. The limit $m_{\mathrm{P}} \rightarrow 0$ does not exist if $\eta_{\mathrm{P}}$ or $\eta_{\mathrm{P}}^{\prime}$ are nonvanishing.

Equipped with arbitrary nonminimal couplings, parametrized by constants $\eta_{i}$, the covariantized action of Pauli-Fierz fields $\Upsilon_{\mu \nu}$ of mass $m_{\Upsilon}$ reads

$$
\begin{aligned}
\hat{S}_{\mathrm{PF}}\left(g, \Upsilon, m_{\Upsilon}^{2}\right)= & S_{\mathrm{PF}}\left(g, \Upsilon, m_{\Upsilon}^{2}\right)+\frac{1}{2} \int \sqrt{-g}\left[\eta_{1} R^{\mu \nu \rho \sigma} \Upsilon_{\mu \rho} \Upsilon_{\nu \sigma}+R^{\mu \nu}\left(\eta_{2} \Upsilon_{\mu \rho} \Upsilon_{\nu}^{\rho}+\eta_{3} \Upsilon_{\mu \nu} \Upsilon\right)\right. \\
& \left.+R\left(\eta_{4} \Upsilon_{\mu \nu} \Upsilon^{\mu \nu}+\eta_{5} \Upsilon^{2}\right)\right]
\end{aligned}
$$


where $\Upsilon=\Upsilon_{\mu \nu} g^{\mu \nu}$. The contribution $\Gamma_{\text {abs }}^{\mathrm{PF}}$ of such fields to the absorptive part is rather involved. We report its high-energy behavior in appendix B, enough to prove that, differently from the case of the Proca fields, no values of the nonminimal couplings make the ultraviolet limit of $\Gamma_{\text {abs }}^{\mathrm{PF}}$ well defined.

In ref. [22] the masses of the matter fields $\Phi$ were set to zero and both $\phi$ and $\chi_{\mu \nu}$ were implicit and quantized as fakeons. This means that the results found there apply to the GFF theory at $r_{\Phi}=0$. Indeed, it is easy to check that formula (4.6) at $r_{\Phi}=0$ agrees with the result of [22], apart from the expression of $\Delta g_{\mu \nu}$, which is much simpler now. The reason behind the change of $\Delta g_{\mu \nu}$ is that the two calculations are done with different classical Lagrangians, (2.1) versus (2.10) or (2.16), related by perturbative field redefinitions. Normally, a change of field variables on the classical action affects the $\Gamma$ functional by modifying the contributions that vanish on the solutions of the field equations. A general method to work out the change of $\Delta g_{\mu \nu}$ directly does exist [25-27] and requires to extend the calculations to the composite fields involved in the transformation.

\section{The fakeon width}

The diagram of figure 1 does not allow us to extract physical quantities for generic values of the center-of-mass energy squared $s=p^{2}$, because the graviton gives gauge-dependent contributions, such as (4.8) and (4.10), which can be turned into cubic corrections by means of field redefinitions. If we want a physical quantity for generic $s$, the diagrams of figure 1 must be accompanied by other diagrams that contribute to the same order and involve triangles (vertex corrections) and boxes. Computations of this type have been done extensively in the standard model [30-32] and can be generalized to the theory of quantum gravity studied here with some effort. However, for the time being, we concentrate on the fakeon widths, which are physical quantities that can be extracted just from the bubble diagrams.

Assume that $s=p^{2}$ is very close to $m_{\chi}^{2}$. Then the leading contributions of the (nonamputated) two-point functions $\left\langle G_{A}(p) G_{B}(-p)\right\rangle$ to $M_{A B}$ are given by $\left\langle\chi_{\mu \nu}(p) \chi_{\rho \sigma}(-p)\right\rangle_{\text {abs }}^{1 \text { loop }}$, which carry a double pole $1 /\left(s-m_{\chi}^{2}\right)^{2}$. The vertex corrections are next-to-leading, and so are the contributions such as $\left\langle h_{\mu \nu}(p) \chi_{\rho \sigma}(-p)\right\rangle_{\text {abs }}^{1 \text { loop }}$, since they give at most simple poles $1 /\left(s-m_{\chi}^{2}\right)$. The gauge-dependent contributions, such as $\left\langle h_{\mu \nu}(p) h_{\rho \sigma}(-p)\right\rangle_{\text {abs }}^{1}$ loop , are nextto-next-to-leading, as are the box corrections.

This means that the coefficient of the double pole must be physical by itself at the fakeon peak. For example, it is straightforward to check that it is gauge independent. Specifically, assuming that the masses of the matter fields $\Phi$ are much smaller than $m_{\chi}$ and $s \sim m_{\chi}$, we find

$$
\left\langle\chi_{\mu \nu}(p) \chi_{\rho \sigma}(-p)\right\rangle_{\mathrm{abs}}^{1 \text { loop }}=C(s) \frac{\kappa^{4}}{8 \pi \zeta^{2}} \frac{s^{2}}{\left(s-m_{\chi}^{2}\right)^{2}} \prod_{\mu \nu \rho \sigma}^{(2)}(p, s)+\mathcal{O}\left(\left(s-m_{\chi}^{2}\right)^{0}\right),
$$

where $\prod_{\mu \nu \rho \sigma}^{(2)}(p, s)$ can be read from (3.6) and

$$
C(s)=C_{m}+C_{\phi}(s), \quad C_{m}=\frac{N_{s}+6 N_{f}+12 N_{v}}{120}, \quad C_{\phi}(s)=\frac{1}{120} \theta\left(1-r_{\phi}\right)\left(1-r_{\phi}\right)^{5 / 2},
$$


with $r_{\phi}=4 m_{\phi}^{2} / s$. Since we are not making assumptions about the mass of $\phi$, we must take $C_{\phi}(s)$ as a function of $s$.

The quantity $C_{m}$ is known as central charge in conformal field theory. By analogy, we can define $C(s)$ as the total central charge and $C_{\phi}(s)$ as the central charge of the massive scalar $\phi$. The function $C_{\phi}(s)$ appearing in (5.2) holds in the GSF theory, where $\phi$ is quantized as a physical field, while $C_{\phi}(s)=0$ in the GFF theory. The central charges of the graviton and the fakeons are identically zero.

If we want, we can include $N_{\mathrm{P}}$ Proca vectors with no nonminimal couplings and small masses $m_{\mathrm{P}}$. Then $C(s)=C_{m}+C_{\mathrm{P}}+C_{\phi}(s)$, with

$$
C_{\mathrm{P}}=\frac{13}{120} N_{\mathrm{P}}
$$

In the presence of Pauli-Fierz fields $\Upsilon_{\mu \nu}$ and when the Proca nonminimal couplings are switched on, the total central charge is a complicated function of $r_{\mathrm{P}}=4 m_{\mathrm{P}}^{2} / s, r_{\Upsilon}=4 m_{\Upsilon}^{2} / s$ and the nonminimal couplings.

Resumming the self-energies, we can obtain the corrected propagators of the graviton multiplet. In particular, at the $\chi$ peak we have the two-point function

$$
\left\langle\chi_{\mu \nu}(p) \chi_{\rho \sigma}(-p)\right\rangle_{s \sim \bar{m}_{\chi}^{2}}=-\frac{i \kappa^{2}}{\zeta} \frac{Z_{\chi}}{s-\bar{m}_{\chi}^{2}+i \bar{m}_{\chi} \Gamma_{\chi}} \prod_{\mu \nu \rho \sigma}^{(2)}(p, s),
$$

where $\bar{m}_{\chi}$ is the corrected $\chi$ mass and $\Gamma_{\chi}$ is the $\chi$ width. We find

$$
\begin{aligned}
& \Gamma_{\chi}=-\frac{\kappa^{2} m_{\chi}^{3}}{8 \pi \zeta} C+m_{\chi} \mathcal{O}\left(\frac{m_{\chi}^{4}}{M_{\mathrm{Pl}}^{4}}\right)=-m_{\chi} \alpha_{\chi} C+m_{\chi} \mathcal{O}\left(\alpha_{\chi}^{2}\right), \\
& Z_{\chi}=1+\mathcal{O}\left(\alpha_{\chi}\right), \quad \bar{m}_{\chi}^{2}=m_{\chi}^{2}\left[1+\mathcal{O}\left(\alpha_{\chi}\right)\right],
\end{aligned}
$$

where $M_{\mathrm{Pl}}$ is the Planck mass, $C=C\left(m_{\chi}^{2}\right)$ and $\alpha_{\chi}=m_{\chi}^{2} / M_{\mathrm{Pl}}^{2}$ is a sort of "fakeon/graviton structure constant".

The negative sign of $\Gamma_{\chi}$ implies that microcausality is violated. We can illustrate this effect in simple terms by means of the Breit-Wigner distribution and its Fourier transform. We have

$$
\frac{i}{E-m+i \frac{\Gamma}{2}} \quad \longrightarrow \quad \operatorname{sgn}(t) \theta(\Gamma t) \exp \left(-i m t-\frac{\Gamma t}{2}\right),
$$

so when $\Gamma<0$ the theta function picks the future instead of the past.

Note that the negative overall sign in front of the propagator (5.3) is consistent with unitarity. Indeed, we find

$$
2 \operatorname{Im}\left[i\left\langle\chi_{\mu \nu}(p) \chi_{\rho \sigma}(-p)\right\rangle_{s \sim \bar{m}_{\chi}^{2}}\right]=-\frac{2 \kappa^{2}}{\zeta} \frac{Z_{\chi} \bar{m}_{\chi} \Gamma_{\chi}}{\left(s-\bar{m}_{\chi}^{2}\right)^{2}+\bar{m}_{\chi}^{2} \Gamma_{\chi}^{2}} \prod_{\mu \nu \rho \sigma}^{(2)}(p, s) \geqslant 0,
$$

in agreement with the optical theorem. In particular, when we take the limit $\Gamma_{\chi} \rightarrow 0^{-}$, we obtain

$$
2 \operatorname{Im}\left[i\left\langle\chi_{\mu \nu}(p) \chi_{\rho \sigma}(-p)\right\rangle_{s \sim \bar{m}_{\chi}^{2}}\right] \underset{\Gamma_{\chi} \rightarrow 0^{-}}{\longrightarrow} \frac{2 \pi \kappa^{2}}{\zeta} Z_{\chi} \delta\left(s-\bar{m}_{\chi}^{2}\right) \prod_{\mu \nu \rho \sigma}^{(2)}(p, s) .
$$


Let us discuss a hypothetical scattering process containing fakeons among the final states. In that case we must take the imaginary part of the zeroth order $\chi_{\mu \nu}$ propagator, which however vanishes because of the quantization prescription (3.5). This means that a process of this type has vanishing cross section and cannot occur. It is impossible to detect $\chi_{\mu \nu}$ "before it decays into something else", which is consistent with calling $\chi_{\mu \nu}$ a "fake particle" and stating that it does not belong to the subspace $V$ of the physical fields. The difference between the peak of a fakeon and the peak of a resonance is that the one of a fakeon is just a geometric shape and no physical particle is associated with it. In some respects, this behavior resembles the one of the "anomalous thresholds" [33]. In particular, the quantity $1 /\left|\Gamma_{\chi}\right|$ cannot be viewed as the lifetime of the fakeon. We could interpret it as the amount of time during which causality is meaningless. More details are given in the next section, where we compare the results of the GFF and GSF theories with those of the theory that has ghosts.

If we repeat the calculation around the $\phi$ peak, under the assumption that the masses of the matter fields are negligible with respect to $m_{\phi}$, we find the width

$$
\Gamma_{\phi}=\frac{\eta_{s}^{2} \kappa^{2} m_{\phi}^{3}}{48 \pi \zeta}=\frac{m_{\phi}}{6} \alpha_{\phi} \eta_{s}^{2}
$$

where $\alpha_{\phi} \equiv m_{\phi}^{2} / M_{\mathrm{Pl}}^{2}$. We expect that $\Gamma_{\phi}$ is much smaller than $\left|\Gamma_{\chi}\right|$, because it is only sensitive to the scalar nonminimal coupling $\eta_{s}$. No sign of microcausality violation is present here, since $\Gamma_{\phi}>0$.

We do not have compelling arguments to predict the values of the masses $m_{\chi}$ and $m_{\phi}$, but it is conceivable that they are smaller than the Planck mass. Taking $m_{\chi} \sim$ $m_{\phi} \sim 10^{11} \mathrm{GeV}$, for definiteness, and assuming the matter content of the standard model $\left(N_{s}=4, N_{f}=45 / 2, N_{v}=12\right)$, we obtain $\alpha_{\chi} \sim 7 \cdot 10^{-17}$ and $\Gamma_{\chi} \sim-16 \mathrm{keV}$. For $m_{\chi} \sim m_{\phi} \sim 10^{12} \mathrm{GeV}$ we would have $\Gamma_{\chi} \sim-16 \mathrm{MeV}$. The dumping factor $\mathrm{e}^{-\Gamma t / 2}$ appearing in formula (5.5) tells us that the violation of causality occurs within time intervals of the order of $4 \cdot 10^{-20} \mathrm{~s}$ for $m_{\chi} \sim 10^{11} \mathrm{GeV}$, in the center-of-mass frame, and $4 \cdot 10^{-23} \mathrm{~s}$ for $m_{\chi} \sim 10^{12} \mathrm{GeV}$. However, the oscillating factor $\mathrm{e}^{-i m t}$ strongly suppresses those effects up to energies of the order of the fakeon mass. Other massive particles with masses smaller than $m_{\chi}$ could be present in nature, besides those contained in the standard model, and make $C$ and $\left|\Gamma_{\chi}\right|$ larger by one or two orders of magnitude.

More effort is necessary to work out physical quantities away from the peaks, such as the cross section $\sigma$ as a function of the center-of-mass energy $\sqrt{s}$. So far we have set the cosmological constant $\Lambda_{C}$ to zero, but it is not difficult to extend the calculations to nonvanishing $\Lambda_{C}$.

\section{Comparison with the Stelle theory}

In this section we compare the results found in the GFF and GSF theories with those that can be obtained in the Stelle GSGh theory, to emphasize the differences and the effects of the ghosts. The GSGh quantization prescription is just made of point (a) of section 3, so $\phi$ is a physical scalar and $\chi_{\mu \nu}$ is a spin-2 ghost. 


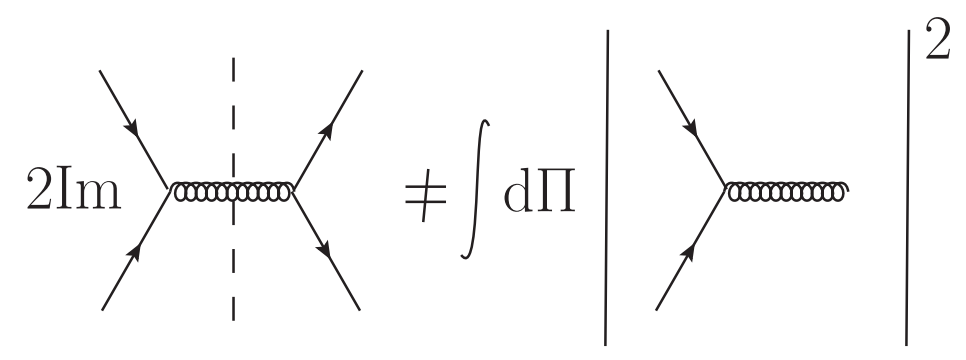

Figure 2. Breakdown of the optical theorem in the GSGh theory.

The absorptive part of the self-energy of the graviton multiplet includes extra bubble diagrams, whose bubbles are made of: (i) two $\chi$ legs, (ii) one $\chi$ leg and one $\phi$ leg, (iii) one $\chi$ leg and one $h$ leg. In total,

$$
\Gamma_{\mathrm{abs}}^{\mathrm{GSGh}}=\Gamma_{\mathrm{abs}}^{\mathrm{GSF}}+\Gamma_{\mathrm{abs}}^{\chi h}+\Gamma_{\mathrm{abs}}^{\chi \phi}+\Gamma_{\mathrm{abs}}^{\chi \chi} .
$$

For the calculations, we use the action (2.17). The corrections can be evaluated straightforwardly, but their expressions are quite lengthy, so we content ourselves with the analysis of the results around the $\chi$ peak. There, $\Gamma_{\text {abs }}^{\chi \chi}$ does not contribute, because its threshold is $s=4 m_{\chi}^{2}$. Similarly, $\Gamma_{\text {abs }}^{\chi \phi}$ has a threshold at $s=\left(m_{\chi}+m_{\phi}\right)^{2}$. Since the $\phi$ mass is presumably not very different from the $\chi$ mass, $\Gamma_{\text {abs }}^{\chi \phi}$ is also negligible at the $\chi$ peak. In the end, only $\Gamma_{\text {abs }}^{\chi h}$ is important. We find formula (5.1) with the modified central charge

$$
C(s)=C_{m}+C_{\phi}(s)+C_{\mathrm{Gh}}(s), \quad C_{\mathrm{Gh}}(s)=-\left(t_{\chi}-1\right) \theta\left(t_{\chi}-1\right),
$$

where $t_{\chi}=s / m_{\chi}^{2}$. The crucial factor $\left(t_{\chi}-1\right)$ in $C_{\mathrm{Gh}}(s)$, brought by formula (A.1), implies that the spin- 2 ghost contribution $C_{\mathrm{Gh}}(s)$ to the central charge is subleading around the peak. The value of $\Gamma_{\chi}$ is still the one of formula (5.4), to the lowest order.

For sufficiently small time intervals, where the ghost is still "alive", we do not have to resum the powers of the width $\Gamma_{\chi}$. Indeed, the Stelle theory admits the process of figure 2, which does not obey the optical theorem. The right-hand side is positive, while the left-hand side is negative, since

$$
\begin{aligned}
2 \operatorname{Im}\left[i\left\langle\chi_{\mu \nu}(p) \chi_{\rho \sigma}(-p)\right\rangle_{0}\right] & =\frac{2 \kappa^{2}}{\zeta} \operatorname{Im}\left[\frac{1}{s-m_{\chi}^{2}+i \epsilon}\right] \prod_{\mu \nu \rho \sigma}^{(2)}(p, s) \\
& =-\frac{2 \pi \kappa^{2}}{\zeta} \delta\left(s-m_{\chi}^{2}\right) \prod_{\mu \nu \rho \sigma}^{(2)}(p, s) \leqslant 0 .
\end{aligned}
$$

The GFF and GSF theories, on the other hand, just give $0=0$ in this case, since the left-hand side has no imaginary part due to the fakeon prescription (3.2), while the right-hand side vanishes since the fakeon is projected away from the physical spectrum. This is another way of saying that the projection in question is consistent.

In some sense, the fakeons can be viewed as "auxiliary fields with nontrivial kinetic terms". They circulate inside the Feynman diagrams, but cannot enter or exit the diagrams. They mediate interactions, but cannot be observed directly. We could even integrate them out (following the rules of the nonanalytic Wick rotation or the average continuation), but the resulting theory would be nonlocal and more difficult to handle. 


\section{Conclusions}

We have studied various aspects of the theory of quantum gravity proposed in ref. [1], after converting its higher-derivative action into an action with two-derivative kinetic terms. The graviton multiplet is made of the fluctuation $h_{\mu \nu}$ of the metric tensor around flat space, a massive scalar $\phi$ and a massive spin-2 field $\chi_{\mu \nu}$. The field $\chi_{\mu \nu}$ is quantized as a fakeon, because its kinetic action has the wrong overall sign. The scalar $\phi$ can be quantized either as a fakeon or a physical particle, which leads to two options, the GFF and GSF theories.

At high energies the nature of $\left\{h_{\mu \nu}, \phi, \chi_{\mu \nu}\right\}$ as a multiplet emerges clearly, the main duty of $\chi_{\mu \nu}$ and $\phi$ being to "escort" the graviton $h_{\mu \nu}$ and wipe away most ultraviolet divergences it creates, to ensure renormalizability. At low energies, both $\chi_{\mu \nu}$ and $\phi$ decouple and the ordinary low energy, nonrenormalizable theory is retrieved.

The action of quantum gravity is strictly renormalizable, which makes it essentially unique (when matter is switched off), because it contains a finite number of parameters and can be quantized in just two physically consistent ways. As in the standard model, the matter sector cannot be predicted from first principles, since it is always possible to add heavy particles and/or fakeons.

We have calculated the absorptive part of the self-energy of the graviton multiplet and used it to compute, among other things, the width $\Gamma_{\chi}$ of the fakeon $\chi_{\mu \nu}$ and the width $\Gamma_{\phi}$ of the scalar $\phi$. The former is negative and proportional to the central charge $C$. The graviton and the fakeons do not contribute to $C$, while the other physical fields give positive contributions. Perturbative unitarity holds, i.e. the optical theorem is satisfied. However, the negative sign of $\Gamma_{\chi}$ shows that the theory predicts the violation of causality for center-of-mass energies larger than the fakeon mass $m_{\chi}$, at distances or time intervals smaller than $1 /\left|\Gamma_{\chi}\right|$. There, the notions of past, present and future lose meaning. Said in different words, the theory implies that causality is not a principle of nature, but an approximation that is practically useful when two events are separated by a time interval longer than $1 /\left|\Gamma_{\chi}\right|$. Since at present quantum gravity is the only interaction of nature that predicts the violation of microcausality, the experimental detection of such effects could be the first sign that gravity is indeed quantized.

The calculations of this paper can be extended to include the vertex corrections and the box contributions, along the lines of analogous computations done in the standard model [30-32], to achieve gauge independence away from the peaks and obtain the complete cross section $\sigma(\sqrt{s})$.

\section{Acknowledgments}

We are grateful to U. Aglietti and D. Comelli for useful discussions.

\section{A Calculations of absorptive parts}

In this appendix we recall how to calculate the absorptive parts of the one-loop diagrams. Consider the integral

$$
I\left(p, m_{1}, m_{2}\right)=\int \frac{\mathrm{d}^{D} k}{(2 \pi)^{D}} S\left(k, m_{1}\right) S\left(p+k, m_{2}\right), \quad S(k, m)=\frac{1}{k^{2}-m^{2}+i \epsilon} .
$$


Using the Feynman parameters and renormalizing the divergence away (because it does not contribute to the absorptive part), we find

$$
I\left(p, m_{1}, m_{2}\right)=-\frac{i}{16 \pi^{2}} \int_{0}^{1} \mathrm{~d} x \ln \left[m_{1}^{2} x+m_{2}^{2}(1-x)-p^{2} x(1-x)-i \epsilon\right] .
$$

The absorptive part is

$$
\begin{aligned}
I_{\text {abs }}\left(p, m_{1}, m_{2}\right) & =-\frac{1}{16 \pi} \int_{0}^{1} \mathrm{~d} x \theta\left(p^{2} x(1-x)-m_{1}^{2} x-m_{2}^{2}(1-x)\right) \\
& =-\frac{1}{16 \pi} \theta\left(p^{2}-\left(m_{1}+m_{2}\right)^{2}\right) \sqrt{1-\frac{\left(m_{1}+m_{2}\right)^{2}}{p^{2}}} \sqrt{1-\frac{\left(m_{1}-m_{2}\right)^{2}}{p^{2}}} .
\end{aligned}
$$

Similarly, we can treat the integrals

$$
\begin{aligned}
I^{\mu_{1} \cdots \mu_{n}}\left(p, m_{1}, m_{2}\right)= & \int \frac{\mathrm{d}^{D} k}{(2 \pi)^{D}} k^{\mu_{1}} \cdots k^{\mu_{n}} S\left(k, m_{1}\right) S\left(p+k, m_{2}\right)=a_{n} p^{\mu_{1}} \cdots p^{\mu_{n}} \\
& +a_{n-2} p^{\left\{\mu_{1}\right.} \cdots p^{\mu_{n-2}} \eta^{\left.\mu_{n-1} \mu_{n}\right\}}+\cdots
\end{aligned}
$$

(the indices between the curly brackets being completely symmetrized) by expanding the results as sums of polynomials built with $p_{\mu}$ and $\eta_{\mu \nu}$, multiplied by constants $a_{i}$. The constants are calculated by contracting with $p_{\mu}$ and $\eta_{\mu \nu}$ and making the replacements

$$
\begin{aligned}
k^{2} & \rightarrow m_{1}^{2}, \quad(p+k)^{2} \rightarrow m_{2}^{2}, \\
p \cdot k & =\frac{1}{2}\left[(p+k)^{2}-p^{2}-k^{2}\right] \rightarrow \frac{1}{2}\left(m_{2}^{2}-m_{1}^{2}-p^{2}\right)
\end{aligned}
$$

in the numerators, which follow from the fact that the tadpoles have no absorptive parts. We get, for example,

$$
\begin{aligned}
& I_{\mathrm{abs}}^{\mu}\left(p, m_{1}, m_{2}\right)=-\frac{p^{\mu}}{2}\left(1+r_{-}\right) I_{\mathrm{abs}}\left(p, m_{1}, m_{2}\right), \\
& I_{\mathrm{abs}}^{\mu \nu}\left(p, m_{1}, m_{2}\right)=\left[\frac{p^{\mu} p^{\nu}}{3}\left(1+r_{-}-r_{2}+r_{-}^{2}\right)-\frac{\eta^{\mu \nu} p^{2}}{12}\left(1-2 r_{+}+r_{-}^{2}\right)\right] I_{\mathrm{abs}}\left(p, m_{1}, m_{2}\right),
\end{aligned}
$$

where $r_{i}=m_{i}^{2} / p^{2}$ and $r_{ \pm}=r_{1} \pm r_{2}$. We can proceed similarly to work out the expressions of all the $I_{\text {abs }}^{\mu_{1} \cdots \mu_{n}}\left(p, m_{1}, m_{2}\right)$. For the calculations of this paper, we just need $n$ from 0 to 4 .

\section{B Contributions of Proca and Pauli-Fierz fields}

Here we collect a few results about the contributions of Proca and Pauli-Fierz fields to the absorptive part (4.12) of the graviton-multiplet self-energy. The nonminimal couplings of the Proca action (4.14) give contributions

$$
\begin{aligned}
P_{\mathrm{P}}^{\mathrm{nm}}(r)= & \frac{N_{\mathrm{P}}}{60} \frac{\eta_{\mathrm{P}}}{r^{2}}\left[\eta_{\mathrm{P}}\left(2+6 r+7 r^{2}\right)-2 r\left(1+13 r+r^{2}\right)\right], \\
Q_{\mathrm{P}}^{\mathrm{nm}}(r)= & \frac{N_{\mathrm{P}}}{144 r^{2}}\left[6 \eta_{\mathrm{P}}^{\prime}\left(6 \eta_{\mathrm{P}}^{\prime}+r\right)\left(4-4 r+3 r^{2}\right)\right. \\
& \left.+\eta_{\mathrm{P}}\left(12 \eta_{\mathrm{P}}^{\prime}+r\right)\left(8-10 r+5 r^{2}\right)+\eta_{\mathrm{P}}^{2}\left(16-24 r+11 r^{2}\right)\right] .
\end{aligned}
$$

The only way to have a smooth ultraviolet limit is by setting $\eta_{\mathrm{P}}=\eta_{\mathrm{P}}^{\prime}=0$. 
In the case of the Pauli-Fierz action (4.15), we report the first terms of the high-energy expansion, given by

$$
\begin{aligned}
P_{\mathrm{PF}}(r) & =\frac{8 N_{\mathrm{PF}}}{135 r^{4}}\left[\left(3-2 \eta_{1}+\eta_{2}\right)^{2}+\frac{r}{4}\left(45-6 \eta_{1}+5 \eta_{2}\right)\left(3-2 \eta_{1}+\eta_{2}\right)+\frac{9}{4} r\right]+\mathcal{O}\left(r^{-2}\right), \\
Q_{\mathrm{PF}}(r) & =\frac{4 N_{\mathrm{PF}}}{81 r^{4}}\left(3-\eta_{1}+2 \eta_{2}+6 \eta_{4}\right)^{2}+\mathcal{O}\left(r^{-3}\right) .
\end{aligned}
$$

We see that if we choose the coefficients $\eta_{i}$ of the nonminimal couplings so that the $\mathcal{O}\left(r^{-4}\right)$ terms vanish, the $\mathcal{O}\left(r^{-3}\right)$ cannot vanish at the same time. Therefore, it is impossible to have a smooth ultraviolet limit, in contrast with what happens in the Proca theory.

Open Access. This article is distributed under the terms of the Creative Commons Attribution License (CC-BY 4.0), which permits any use, distribution and reproduction in any medium, provided the original author(s) and source are credited.

\section{References}

[1] D. Anselmi, On the quantum field theory of the gravitational interactions, JHEP 06 (2017) 086 [http://renormalization.com/17a3/] [arXiv:1704.07728] [INSPIRE].

[2] D. Anselmi, Fakeons And Lee-Wick Models, JHEP 02 (2018) 141 [http://renormalization.com/18a1/] [arXiv: 1801.00915] [INSPIRE].

[3] T.D. Lee and G.C. Wick, Negative Metric and the Unitarity of the S Matrix, Nucl. Phys. B 9 (1969) 209 [inSPIRE].

[4] T.D. Lee and G.C. Wick, Finite Theory of Quantum Electrodynamics, Phys. Rev. D 2 (1970) 1033 [InSPIRE].

[5] R.E. Cutkosky, P.V. Landshoff, D.I. Olive and J.C. Polkinghorne, A non-analytic S matrix, Nucl. Phys. B 12 (1969) 281 [InSPIRE].

[6] B. Grinstein, D. O'Connell and M.B. Wise, Causality as an emergent macroscopic phenomenon: The Lee-Wick O(N) model, Phys. Rev. D 79 (2009) 105019 [arXiv:0805.2156] [inSPIRE].

[7] D. Anselmi and M. Piva, A new formulation of Lee-Wick quantum field theory, JHEP 06 (2017) 066 [http://renormalization.com/17a1/] [arXiv:1703.04584] [inSPIRE].

[8] D. Anselmi and M. Piva, Perturbative unitarity of Lee-Wick quantum field theory, Phys. Rev. D 96 (2017) 045009 [http://renormalization.com/17a2/] [arXiv: 1703. 05563] [INSPIRE].

[9] S.B. Giddings, The Boundary $S$ matrix and the AdS to CFT dictionary, Phys. Rev. Lett. 83 (1999) 2707 [hep-th/9903048] [INSPIRE].

[10] V. Balasubramanian, S.B. Giddings and A.E. Lawrence, What do CFTs tell us about Anti-de Sitter space-times?, JHEP 03 (1999) 001 [hep-th/9902052] [INSPIRE].

[11] J. Bros, H. Epstein, M. Gaudin, U. Moschella and V. Pasquier, Triangular invariants, three-point functions and particle stability on the de Sitter universe,

Commun. Math. Phys. 295 (2010) 261 [arXiv:0901.4223] [INSPIRE].

[12] E.T. Akhmedov and P.V. Buividovich, Interacting Field Theories in de Sitter Space are Non-Unitary, Phys. Rev. D 78 (2008) 104005 [arXiv: 0808.4106] [INSPIRE].

[13] E.T. Akhmedov, Lecture notes on interacting quantum fields in de Sitter space, Int. J. Mod. Phys. D 23 (2014) 1430001 [arXiv:1309.2557] [InSPIRE]. 
[14] D. Marolf, I.A. Morrison and M. Srednicki, Perturbative S-matrix for massive scalar fields in global de Sitter space, Class. Quant. Grav. 30 (2013) 155023 [arXiv:1209.6039] [INSPIRE].

[15] K. Hinterbichler and M. Saravani, Stückelberg approach to quadratic curvature gravity and its decoupling limits, Phys. Rev. D 93 (2016) 065006 [arXiv: 1508.02401] [InSPIRE].

[16] J. Julve and M. Tonin, Quantum Gravity with Higher Derivative Terms, Nuovo Cim. B 46 (1978) 137 [InSPIRE].

[17] E.S. Fradkin and A.A. Tseytlin, Renormalizable asymptotically free quantum theory of gravity, Nucl. Phys. B 201 (1982) 469 [INSPIRE].

[18] I.G. Avramidi and A.O. Barvinsky, Asymptotic freedom in higher derivative quantum gravity, Phys. Lett. B 159 (1985) 269 [INSPIRE].

[19] N. Ohta, R. Percacci and A.D. Pereira, Gauges and functional measures in quantum gravity II: Higher derivative gravity, Eur. Phys. J. C 77 (2017) 611 [arXiv:1610.07991] [INSPIRE].

[20] A. Salvio and A. Strumia, Agravity, JHEP 06 (2014) 080 [arXiv:1403.4226] [INSPIRE].

[21] A. Salvio and A. Strumia, Agravity up to infinite energy, Eur. Phys. J. C 78 (2018) 124 [arXiv: 1705.03896] [INSPIRE].

[22] D. Anselmi and M. Piva, The Ultraviolet Behavior of Quantum Gravity, JHEP 05 (2018) 027 [http://renormalization.com/18a2/] [arXiv: 1803.07777] [INSPIRE].

[23] S. Coleman, Acausality, in Erice 1969 Ettore Majorana School On Subnuclear Phenomena, Academic Press, New York (1970).

[24] K.S. Stelle, Renormalization of Higher Derivative Quantum Gravity, Phys. Rev. D 16 (1977) 953 [INSPIRE].

[25] D. Anselmi, A General Field-Covariant Formulation Of Quantum Field Theory, Eur. Phys. J. C 73 (2013) 2338 [http://renormalization.com/12a1/] [arXiv:1205.3279] [INSPIRE].

[26] D. Anselmi, A Master Functional For Quantum Field Theory, Eur. Phys. J. C 73 (2013) 2385 [http://renormalization.com/12a2/] [arXiv:1205.3584] [INSPIRE].

[27] D. Anselmi, Master Functional And Proper Formalism For Quantum Gauge Field Theory, Eur. Phys. J. C 73 (2013) 2363 [http://renormalization.com/12a3/] [arXiv:1205.3862] [INSPIRE].

[28] R.E. Cutkosky, Singularities and discontinuities of Feynman amplitudes, J. Math. Phys. 1 (1960) 429 [INSPIRE].

[29] M.J.G. Veltman, Unitarity and causality in a renormalizable field theory with unstable particles, Physica 29 (1963) 186 [INSPIRE].

[30] W.L. van Neerven, Dimensional Regularization of Mass and Infrared Singularities in Two Loop On-shell Vertex Functions, Nucl. Phys. B 268 (1986) 453 [INSPIRE].

[31] H. Dorn, Renormalization of Path Ordered Phase Factors and Related Hadron Operators in Gauge Field Theories, Fortsch. Phys. 34 (1986) 11 [InSPIRE].

[32] M. Consoli, W.F.L. Hollik and F. Jegerlehner, Electroweak radiative corrections for $Z$ physics, in $Z$ physics at LEP 1, Vol. 1: Standard physics, G. Altarelli, R. Kleiss and C. Verzegnassi eds., CERN-1989-008-V-1.

[33] R.J. Eden, P.V. Landshoff, D.I. Olive and J.C. Polkinghorne, The Analytic S-Matrix, Cambridge University Press, Cambridge, U.K. (1966) [INSPIRE]. 